\title{
Influence of the physico-chemical form on americium biodistribution after wound contamination: re-analysis of experimental data using STATBIODIS
}

\author{
Stephanie Lamart ${ }^{1, *}$, Anne Van der Meeren ${ }^{1}$, Sophie Dubois ${ }^{2}$, Solveig Bibard ${ }^{1}$, Ana Paula \\ Serond $^{3}$, Jaime Angulo ${ }^{1}$, and Nina M Griffiths ${ }^{1}$ \\ ${ }^{1}$ Laboratoire de Radio Toxicologie, CEA, Université Paris-Saclay, 91297 Arpajon, France \\ ${ }^{2}$ Anastats, 37220 Rilly sur Vienne, France \\ ${ }^{3}$ Orano, 92400 Courbevoie, France
}

\section{Context}

Aging of nuclear fuel and decommissioning of nuclear power plants may give rise of wound contaminations with complex mixtures of actinides. To understand better the actinide behaviour in these conditions and to evaluate the efficacy of decorporation protocols, a rodent model of wound contamination has been developed in the laboratory using different wound types, actinides, and physico-chemical forms [1]. More recently, a statistical tool, described in [2] and hereinafter named STATBIODIS, has been developed to facilitate the analysis of biodistribution data from internal contamination experiments. Hence, STATBIODIS allows re-analyses of data from experiments using different contamination conditions and/or carried out at different time periods.

The present study aimed at evaluating the influence of the physico-chemical form and of the presence of plutonium $(\mathrm{Pu})$ in the contaminant, on the biodistribution of americium (Am) after wound contamination.

\section{Material and methods}

Retention and excretion data were collected from several series of wound experiments using $\mathrm{Am}$ alone or in presence of $\mathrm{Pu}$, and in two physico-chemical forms (nitrate or MOX: mixed oxide of uranium and $\mathrm{Pu}$ isotopes, also containing Am-241 as decay product of $\mathrm{Pu}-$ 241). Results of activity measurements in 189 tissues and 161 urine samples were organized in a standardized table format, which built up to 350 lines of data. Descriptive statistics analysis was carried out for each experimental group using STATBIODIS. Am tissue retention and urinary excretion of each animal were analysed as percentage of Am administered activity. To compare Am relative systemic biodistribution between the selected experimental conditions, skeleton to liver activity ratios were also computed. Statistical tests were used to evaluate the influence of the physico-chemical form and of the

*Corresponding author: stephanie.lamart@cea.fr 
presence of plutonium in the contaminant on Am tissue retention and urinary excretion. Non parametric tests essentially adapted to paired data, such as the Wilcoxon test, were applied. Significance level of 0.05 was chosen for bilateral tests and 0.025 for unilateral tests. The false discovery rate correction was applied for multiple comparisons.

\section{Results}

The $24 \mathrm{~h}$ urinary excretion of Am was significantly greater for Am nitrate than for Am from MOX from day 1 to 7 . In addition, excretion data were fitted with sums of exponential terms, which showed a greater excretion rate for Am nitrate. Kidney, skeleton and liver retentions were greater for Am nitrate than for Am from MOX at day 1 and also at day 7 after contamination. Skeleton to liver activity ratios were not significantly different between Am nitrate and Am from MOX seven days after contamination. Hence, Am relative biodistribution between skeleton and liver was independent of the initial physicochemical form, whereas there was overall a greater retention in systemic tissues for Am nitrate than for Am from MOX.

One day after wound contamination, no difference was found between Am nitrate and Am nitrate mixed with $\mathrm{Pu}$ in terms of urinary excretion, tissue retention (blood, lymph node, kidney, liver) and skeleton to liver ratio. Overall, the presence of $\mathrm{Pu}$ did not modify Am biodistribution after contamination with Am nitrate mixed with $\mathrm{Pu}$ after the first 24 hours.

\section{Conclusion}

In this work, experimental data were re-used to evaluate the influence of the physicochemical form and of the presence of $\mathrm{Pu}$ on the $\mathrm{Am}$ biodistribution after wound contamination. Although not all contamination conditions were available across collected data, this re-analysis of biodistribution data using STATBIODIS provided answers to new questions without carrying out any further animal experiments, which is in line with the concept of the 3Rs (Reduce, Refine, Replace) of ethical animal research.

Results presented here were obtained in the frame of the Orano-CEA collaboration agreement.

\section{References}

1. N.M. Griffiths, J.C. Wilk, M.C. Abram, D. Renault, Q. Chau, N. Helfer, C. Guichet, and A. Van der Meeren, Health Phys. 103(2): p. 187-94 (2012).

2. S. Lamart, N.M. Griffiths, N. Tchitchek, J.F. Angulo, and A. Van der Meeren, J Radiol Prot. 37(1): p. 296-308 (2017). 